\title{
Alterações topográficas do disco óptico provocadas pela ing'estão oral de glicerina
}

\author{
Effects of ingestion ofglycerol on optic disc topography
}

\author{
Marcelo Teixeira Nicolela ${ }^{1}$ \\ Roberto Murillo L de Souza Carvalho ${ }^{2}$ \\ Sylvia Pasternak ${ }^{3}$ \\ Adriana Borges da Silva ${ }^{4}$ \\ Remo Susanna Júnior ${ }^{5}$
}

\section{RESUMO}

Objetivo: Verificar os efeitos na topografia do disco óptico após a ingestão de glicerina, uma droga que pode atuar significativamente tanto na pressão intra-ocular (Po) como na pressão do líquido cérebro-raquidiano (PLCR). Métodos: Foram estudados 14 pacientes glaucomatosos recentemente diagnosticados com pressão intra-ocular acima de $25 \mathrm{mmHg}$, sem uso prévio de terapia hipotensora. Foram analisados parâmetros topográficos com o "Heildelberg Retina Tomograph" antes e após a ingestão de glicerina. Resultados: A administração de glicerina produziu uma redução significante da pressão intra-ocular de 28,2\% (95\% IC de 20,7 a 32,1\%). Não foram observadas alterações estatisticamente significantes após ingestão de glicerina nos seguintes parâmetros topográficos: área da escavação, volume da escavação, área da rima, volume da rima, medida da forma da escavação, profundidade média da escavação e profundidade máxima da escavação. Conclusões: Nenhuma mudança significante foi observada nos parâmetros topográficos com o "Heildelberg Retina Tomograph" após a ingestão de glicerina.

Descritores: Pressão intra-ocular; Disco óptico/efeitos de drogas; Disco óptico/anatomia \& histologia; Glicerol/administração \& dosagem; Glaucoma; Tomografia

\section{INTRODUÇ̃̃̃O}

Alguns autores acreditam que a redução da pressão intra-ocular (Po) acima de $30 \%$ em pacientes adultos glaucomatosos promovida por cirurgia filtrante ou por drogas hipotensoras possa promover diminuição da escavação, e que isto seja um sinal de bom prognóstico ${ }^{(1-5)}$.

Duas forças antagonistas podem atuar na lâmina cribiforme do disco óptico, determinando sua posição: a Po e a pressão do líquido cérebro-raquidiano (PLCR), a qual determina a pressão na porção retrolaminar do disco óptico ${ }^{(6-7)}$.

Recentemente, tem sido utilizados aparelhos como tomógrafo de retina para análise topográfica do disco óptico e retina peripapilar. Alguns estudos demonstraram que um desses aparelhos, o "Heidelberg Retina Tomograph" (HRT), consegue detectar mudanças topográficas no disco óptico e na retina peripapilar após redução da pressão intra-ocular $(\mathrm{Po})^{(2-5)}$.

O objetivo do nosso estudo foi verificar os efeitos na topografia do disco óptico de pacientes glaucomatosos recentemente diagnosticados após a ingestão de glicerina, uma droga que pode atuar significativamente tanto na Po como na PLCR.

\section{MÉTODOS}

Critérios de inclusão: pacientes com glaucoma de diagnóstico recente, 
apresentando Po acima de $25 \mathrm{mmHg}$, sem uso prévio de terapia hipotensora; todos pacientes tinham evidências de dano no disco óptico e defeito de campo visual (degrau nasal, defeitos arqueados superior e inferior ou campo tubular) e pacientes com glaucoma primário e secundário foram incluídos. Pacientes com diabetes, nefropatia ou cardiopatia foram excluídos. Uma carta de consentimento assinada foi obtida de cada paciente e o estudo foi aceito pela Comissão de Ética do Hospital.

Após uma série inicial de três imagens de boa qualidade obtidas com o HRT, os pacientes receberam glicerina por via oral ( $1 \mathrm{~g} / \mathrm{kg}$ de peso corporal). Após 45 minutos, novas séries de imagens foram obtidas. A Po foi medida logo após a série inicial de três imagens com o uso do Tonopen e após 45 minutos da ingestão de glicerina.

Imagens com o HRT foram obtidas com as pupilas com pelo menos $3 \mathrm{~mm}$. Foi realizada, utilizando-se cada série de três imagens, uma imagem média, usando o software do equipamento. Uma imagem diferencial, subtraindo-se a imagem média final (pós-glicerina) da inicial (pré-glicerina), também foi criada com o software do aparelho.

Foram analisados parâmetros pré e pós glicerina, incluindo: área da escavação, volume da escavação, área da rima, volume da rima, medida da forma da escavação, profundidade média da escavação, profundidade máxima da escavação, espessura média da camada de fibras nervosas da retina (CFNR) e área de corte transversal da CFNR.

Foi utilizando o programa estatístico SPSS. Valores de $\mathrm{p} \leq 0,05$ foram considerados significantes.

\section{RESULTADOS}

Entre os pacientes estudados, 10 (71\%) eram do sexo masculino e 4 (29\%) eram do sexo feminino. A idade média dos pacientes foi de 51 anos. Em relação ao tipo de glaucoma, 10 (71\%) eram do tipo primário de ângulo aberto, 1 (7\%) era do tipo primário de ângulo fechado, 2 (14\%) eram do tipo secundário e $1(7 \%)$ do tipo juvenil. (Tabela 1 )

A administração de glicerina produziu uma redução significante da Po de 28,2\% (95\% IC de 20,7 a 32,1\%).

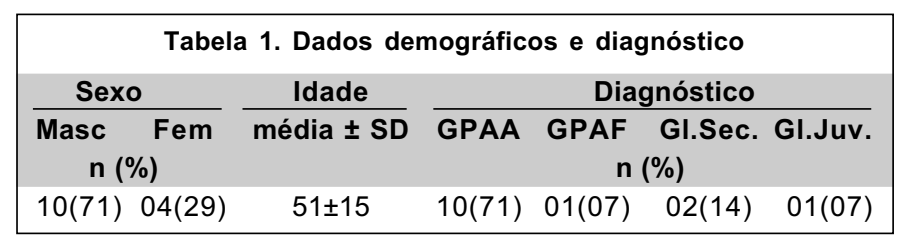

Nenhuma mudança significativa foi observada após ingestão de glicerina nos seguintes parâmetros: área da escavação, volume da escavação, área da rima, volume da rima, medida da forma da escavação, profundidade média da escavação e profundidade máxima da escavação. (Tabela 2)

Houve uma significante redução da espessura média da camada de fibras nervosas da retina (CFNR) e área de corte transversal da CFNR após ingestão de glicerina $(p=0,03)$. Estes resultados não são dependentes do plano de referência, pois não se observou mudança significativa do plano de referência após a glicerina. Além disso, analisamos todos os parâmetros topográficos utilizando um plano de referência fixo ( $320 \mu \mathrm{m}$ posterior ao anel de referência), e obtivemos os mesmos resultados.

Nenhuma correlação foi encontrada entre a redução percentual da Po e a mudança percentual de qualquer parâmetro topográfico.

A análise da imagem diferencial não mostrou mudança em 10 casos $(71 \%)$ e possível deslocamento posterior (piora) do disco óptico em 4 casos (29\%).

\section{DISCUSSÃO}

A ingestão de glicerina produziu uma redução significante da Po. Apesar da redução da Po, nenhuma alteração dos parâmetros topográficos do disco óptico foi observada. Estes resultados diferem de relatos prévios de melhora dos parâmetros topográficos após redução da Po produzida por cirurgia, betaxolol ou apraclonidina ${ }^{(2-4)}$.

As possíveis explicações para esta discrepância são: o estudo presente lida com pacientes sem tratamento prévio, os quais

\begin{tabular}{|c|c|c|c|c|}
\hline $\begin{array}{l}\text { Parâmetros } \\
\text { topográficos e Po }\end{array}$ & Medida inicial & Medida após glicerina & Mudança \% média & Valor de $p$ \\
\hline Po $(\mathrm{mmHg})$ & $36,0 \pm 9,5$ & $26,4 \pm 9,8$ & $28,2 \pm 10,4$ & 0,000 \\
\hline Área da escavação $\left(\mathrm{mm}^{2}\right)$ & $1,42 \pm 0,83$ & $1,45 \pm 0,83$ & $2,9 \pm 7,4$ & 0,27 \\
\hline Área da rima neural $\left(\mathrm{mm}^{2}\right)$ & $1,11 \pm 0,43$ & $1,08 \pm 0,46$ & $-3,5 \pm 11,3$ & 0,30 \\
\hline Vol. da escavação $\left(\mathrm{mm}^{3}\right)$ & $0,74 \pm 0,71$ & $0,77 \pm 0,74$ & $4,9 \pm 12,8$ & 0,25 \\
\hline Prof. máx. de esc. (mm) & $0,92 \pm 0,34$ & $0,95 \pm 0,34$ & $7,1 \pm 25,5$ & 0,20 \\
\hline Medida do formato da esc. & $-0,07 \pm 0,19$ & $-0,08 \pm 0,18$ & $3,7 \pm 77,2$ & 0,11 \\
\hline Prof. méd. da CFNR (mm) & $0,21 \pm 0,09$ & $0,18 \pm 0,1$ & $-15,6 \pm 27,3$ & 0,03 \\
\hline Área corte trans. $\left(\mathrm{mm}^{2}\right)$ & $1,14 \pm 0,43$ & $0,98 \pm 0,47$ & $-15,5 \pm 27,2$ & 0,03 \\
\hline
\end{tabular}


tinham geralmente pressões muito altas (média de $36 \mathrm{mmHg}$ ), diferentemente dos estudos citados anteriormente, que utilizaram pacientes que estavam em tratamento para glaucoma. Outra possibilidade é de que a glicerina, na dose usada neste estudo, pode diminuir não somente a Po mas também a PLCR. Portanto, diferentemente dos estudos prévios, é possível que ambos os componentes do gradiente de pressão tecidual translaminar estejam sendo afetados. Esse fato poderia explicar a não ocorrência de alterações no posicionamento da lâmina cribiforme e outras estruturas do disco óptico observadas em nosso estudo, se assumirmos que ambos fatores (Po e PLCR) foram afetados igualmente pela ingestão de glicerina. Em quatro dos nossos pacientes, houve na verdade uma piora significante dos parâmetros topográficos, significando um deslocamento posterior das estruturas do disco óptico. É possível, que nesses pacientes, a glicerina provocou uma mudança proporcional mais acentuada na PLCR do que na Po. Chauhan e $\operatorname{cols}^{(7)}$ demonstrou experimentalmente que a posição da lâmina cribiforme parece ser mais dependente da PLCR do que da Po.

Como a PLCR não foi medida, por razões óbvias no nosso estudo, as considerações acima são apenas expeculativas. Seria extremamente interessante a validação dessas hipóteses através de estudo experimental.

Interessantemente, a retina peripapilar mostrou um comportamento diferente do disco óptico. Houve uma diminuição da espessura média da CFNR e área de corte transversal da CFNR. Estes resultados são difíceis de explicar, mas mostram comportamento diferente da camada de fibras nervosas e das estruturas do disco óptico.

Este estudo sugere que os movimentos na lâmina cribiforme são guiados não apenas pela Po. Este achado está de acordo com estudos experimentais prévios ${ }^{(6-7)}$. É possível que o gradiente de pressão tecidual translaminar seja isoladamente mais importante que a Po na determinação da posição da lâmina cribiforme. Como o deslocamento posterior da lâmina tem sido implicado no desenvolvimento de dano mecânico dos axônios no glaucoma, isto pode ter importância patogênica.

Assim sendo, podemos concluir que após a ingestão oral de glicerina, nenhuma mudança significante foi observada nos parâmetros topográficos do disco óptico com o HRT.

\section{ABSTRACT}

Purpose: To verify the effects on optic disc topography of newly diagnosed glaucoma subjects after ingestion of glycerol, a drug that causes major changes in both the intra-ocular pressure (IOP) and cerebrospinal fluid pressure (CSFP). Methods: We studied 14 patients presenting with intra-ocular pressure over $25 \mathrm{mmHg}$, without previous use of hypotensive therapy. Results: Glycerol produced a significant intra-ocular pressure average reduction of $28.2 \%$ (95\% CI of 20.7 to $32.1 \%$ ). Despite the obtained intraocular pressure reduction, no significant change was observed in any evaluated topographic parameter (cup area and volume, rim area and volume, maximal and mean cup depth). Conclusions: Overall, no significant change in the topographic parameters, using the "Heildelberg Retina Tomograph", was observed after ingestion of glycerol.

Keywords: Intra-ocular pressure; Optic disc/drug effects; Optic disc/anatomy \& histology; Glycerol/administration \& dosage; Glaucoma; Tomography

\section{REFERÊNCIAS}

1. Spaeth GL. Reversible changes in the optic disc and visual field in glaucoma. Curr Opin Ophthalmol 1994;5:36-45.

2. Irak I, Zangwill L, Garden V, Shakiba S, Weinreb RN. Change in optic disk topography after trabeculectomy. Am J Ophthalmol 1996;122:690-5.

3. Raitta C, Tomita G, Vesti E, Harju M, Nakao H. Optic disc topography before and after trabeculectomy in advanced glaucoma. Ophthalmic Surg Lasers 1996;27:349-54

4. Weinreb RN, Dreher AW. Effect of lowering intra-ocular pressure on optic nerve head topography. In: Nasemann JE, Burk ROW, editors. Scanning laser ophthalmoscopy and topography. Munich: Quitessenz; 1990. p.193-6.

5. Orr AC, Chauhan BC, Levy DM. Effect of acute intra-ocular pressure reduction on optic nerve head topography. A comparison between confocal scanning laser tomography and conventional photography [abstract]. Invest Ophthalmol Vis Sci 1997;38:S830.

6. Morgan WH, Yu DY, Cooper RL, Alder VA, Cringle SJ, Constable IJ. The influence of cerebrospinal fluid pressure on the lamina cribrosa tissue pressure gradient [commented on Invest Ophthalmol Vis Sci 1995;38:163-4]. Invest Ophthalmol Vis Sci 1995;36:1163-72.

7. Chauhan BC, Morgan WH, House PH, Yu D-H. Effects of intra-ocular pressure and cerebrospinal fluid pressure modulation on optic nerve head topography [abstract]. Invest Ophthalmol Vis Sci 1996;37:S664.

\section{Ao enviar um artigo para publicação,} leia ATENTAMENTE as instruções para autores, constante no final de cada fascículo. 